\title{
IMPLANTAÇÃO DE GESTÃO DE PROCESSOS DE NEGÓCIOS (BPM) NAS ORGANIZAÇÕES.
}

\author{
Autor: Daniel Geraldo Rodrigues ${ }^{1}$ \\ Coautor: Luiz Ricardo Mangili ${ }^{2}$
}

\section{RESUMO}

O Foco desta pesquisa é identificar quais os fatores implicam em barreiras que um gerente de projetos pode encontrar para implementar a disciplina de gerenciamento de processos de negócio (BPM) dentro das organizações. O objetivo é conhecer as vantagens que o BPM pode proporciona, conhecer suas características e as dificuldades encontradas para sua implantação. Para isso foi feito uma busca aprofundada na literatura em artigos que abordaram diversos estudos de casos, de diversas localidades, os dados foram coletados e posteriormente utilizados para formular um questionário, onde foi apresentado para dois especialistas da região, que contribuíram com suas práticas e experiências vividas implantado mudanças de organização funcional para organização suportada a processos (BPM) em várias empresas da região.

Palavras-chave: Gestão de projetos. Gerenciamento de processos de negócios (BPM). Barreiras.

\section{ABSTRACT}

The focus of this research is to identify which factors imply the barriers that a project manager may encounter to implement the business process management (BPM) discipline within organizations. The objective is to know the advantages that BPM can provide, to know its characteristics and the difficulties encountered for its implementation. In order to do so, a thorough search was conducted in the literature in articles that dealt with several case studies, from different locations, the data were collected and later used to formulate a questionnaire, where it was presented to two experts from the region who contributed their practices and lived experiences implemented

\footnotetext{
1 Graduado em Administração de Empresas, MBA Gestão de Projetos
} 
changes of functional organization to organization supported to processes (BPM) in several companies of the region.

Keywords: Project management. Business process management (BPM). Barriers.

\section{INTRODUÇÃO}

Diversos são os obstáculos quando se depara com a necessidade de mudanças, seja ela no convívio família ou profissional. O ser humano encara mudança como uma barreira a ser superada. O mundo sempre esteve em constantes mudanças, o que está acontecendo na atualidade é que essas mudanças estão ocorrendo num ritmo cada vez mais acelerado (GONÇALVES; CAMPOS, 2012).

Com o crescimento da gestão de projetos em todo o mundo, a aceitação entre as organizações e seus profissionais é relativamente boa, porém existem barreiras a ser superadas, muitas vezes o projeto só é aceito da boca pra fora, pois os executivos e a alta gerência criam barreiras significativas, fazendo com que os projetos não desenvolvam adequadamente, com isso os profissionais que estão engajados na elaboração sofrem frustrações no decorrer de suas atividades, que podem originar o mau controle de custos, mau fluxo de informações, excesso de dependências externas resultando em atrasos do cronograma. Essas barreiras existem em todo o mundo, entretanto nos países emergentes elas são mais evidentes, barreiras culturais, relativas a gestão de projetos, políticas relacionadas a status são algumas delas, entre outras (KERZNER, 2016). Para atacar um problema primeiramente é preciso conhecê-lo. A implantação de uma metodologia para superar essas barreiras torna-se imprescindível para um desempenho positivo do projeto.

Uma das formas de minimizar os riscos de insucesso na implantação de um projeto é conhecendo as dificuldades que podem ou não ocorrer durante este processo. Na implantação de um projeto, é relevante não somente conhecer os benefícios e melhorias que ele pode proporcionar, também é importante conhecer suas complexidades. Muitos materiais desenvolvidos sobre BPM falam de seus atributos, porém esta pesquisa dedica-se a relatar sobre as possíveis barreiras que os gerentes de projetos encontram para implantar um projeto de BPM nas mais diversas organizações. O propósito é pesquisar a respeito das experiências de projetos de implantação da disciplina BPM nas organizações e seu grau de complexidade, com o objetivo de encontrar materiais que possam trazer informações decorrentes desse assunto. Como identificar as principais dificuldades na implantação de um projeto de 
BPM nas organizações. O que esses dados podem auxiliar no desenvolvimento de um projeto.

Ao analisar diferentes materiais, o autor depara-se com variáveis que podem influenciar no julgamento das diversas barreiras, como diz Chiavenato (2003). Sobre a perspectiva em que as organizações são administradas num contexto ambiental mais amplo, visualizar cada atividade cada tarefa é necessário, pois nada é absoluto tudo é relativo, partindo da corrente mais recente, a teoria da contingência que abrange as circunstancias tecnológicas e ambientais das organizações.

Além de enfrentar profundos problemas que as fizeram buscar as potenciais vantagens de uma solução BPM, as organizações se deparam com uma variedade de outros problemas, algumas vezes inesperados ao embarcar num projeto de implantação de BPM, ao mesmo tempo em que pesquisas são insuficientes para auxiliar neste desafio (INDULSKA et al., 2006). A pesquisa busca contextualizar os fatores que podem implicar em possíveis barreiras para implantar BPM.

\section{REFERENCIAL TEÓRICO}

\section{Conceitos de Projetos}

O PMI (2014) trata projeto como um esforço temporário realizado na criação de um produto ou serviço, com início e término definido, é entendido que o projeto alcance seu objetivo, quando entrega o produto ou serviço combinado, quando as partes interessadas pedem seu encerramento, quando seus objetivos não serão atingidos ou quando o objetivo se torna insignificante. Várias organizações desenvolvem projetos utilizando como base o guia do conhecimento em gerenciamento de projetos (PMBOK), as características e as necessidades de cada organização faz com que desenvolvam suas próprias metodologias de gerenciamento de projetos, o guia é referência, pois reúne as mais abrangentes práticas reconhecidas mundialmente.

Projetos auxiliam as empresas a se adequarem aos novos paradigmas da administração de empresas, visando se adaptar à competitividade do mercado que é cada vez maior. A criação do projeto conta com início e fim predefinido, ele é conduzido de acordo com as especificações preestabelecidas. Para que essas atividades sejam efetivadas, diversos esforços como planejamento e disciplina são empreendidos no decorrer desse período (CAMARGO JUNIOR; PIRES. 2017). 
O conceito mais comum utilizado na definição de um projeto são os resultados exclusivos num determinado período. Para Mansur (2007), os projetos são mudanças estruturadas, que quando implementadas com sucesso atendem as necessidades do negócio, proporcionando melhora na produtividade, redução dos riscos, ao mesmo tempo direcionando as ameaças e as oportunidades através da otimização dos processos.

Para se tornarem competitivas, as organizações necessitam constantemente se adequar as evoluções tecnológicas e utilizarem de estratégias e métodos que prometem proporcionar melhorias em seus negócios. Neste contexto, o profissional de gerenciamento de projetos tem chances maiores de sucesso se constantemente buscar um diferencial para se destacar num mercado cada vez mais competitivo (MEDEIROS, 2011). O gerenciamento de projetos tornou-se predominante nas estratégias empresariais, pois a competição entre elas faz com que busquem as meIhores práticas de gerenciamento para se sobressaírem.

\section{GERENCIAMENTO DE PROCESSOS DE NEGÓcIOS Business Process Mana- gement - BPM}

Gestão por processo de negócio (Business Process Management - BPM) segundo (ABPM, 2013, p 40)

Uma disciplina gerencial que integra estratégias e objetivos de uma organização com expectativas de clientes, por meio do foco em processos ponta a ponta. BPM engloba estratégias, objetivos, cultura, estruturas organizacionais, papéis, políticas, métodos e tecnologias para analisar, desenhar, implementar, gerenciar desempenho, transformar e estabelecer a governança de processos.

A falta de publicações que esclareça os termos que compõe nomenclatura básica de BPM, particularmente como, gerenciamento de processos de negócios, processos de negócios e contraste com gerenciamento de fluxo de trabalho e processos de negócios que Ko (2009), orienta começar abordando a visão geral dos fundamentos do BPM.

Efetivamente BPM é entendido como a prioridade principal das organizações que querem sobreviver aos atuais mercados competitivos, ele abrange procedimentos, técnicas e ferramentas que visam apoiar projetos, a difusão, gestão e análise de processos de negócios (INDULSKA et al., 2006). Há evidencias de que empresas 
que empregam uma disciplina alinhada a processos ágeis, e que buscam a melhoria contínua, ao mesmo tempo em que alinha sua cultura organizacional par se adaptar as novas mudanças é capaz de dar uma resposta mais rápida para o mercado.

Além de enfrentar profundos problemas que as fizeram buscar as potenciais vantagens de uma solução BPM, as organizações se deparam com uma variedade de outros problemas, algumas vezes inesperados ao embarcar num projeto de implantação de BPM, ao mesmo tempo em que pesquisas são insuficientes para auxiliar neste desafio (INDULSKA et al., 2006). A identificação e compreensão desses problemas são de vital importância, no entendimento que num projeto, trabalhar com medidas de prevenção traz vantagem, de forma que riscos podem ser minimizados, mitigados, com isto proporcionando diminuição na probabilidade de atrasos e maiores custos.

De um lado BPM visa processos de negócios operacionais sem o uso de novas tecnologias, tendo em vista que o escopo é amplo, que vai da análise e automação de processos até a gestão e organização do trabalho. Por outro lado, ele é constantemente associado com software com o objetivo de gerenciar controlar e suportar os processos operacionais (VAN DER AALST, 2013). Com o propósito de encarar tal desafio a tecnologia da informação lançou sistemas de software genéricos capaz de suportar o gerenciamento de processos operacionais (KO, 2009). Neste contexto plataformas tecnológicas denominadas BPMS (Business Process Management System), prometem integrar a gestão dos processos de negócios visando promover maior agilidade e flexibilidade nas operações (ENOKI, 2006). Após pesquisar muitos autores, entende-se que BPM está alinhado à conhecimentos de processos de negócios e tecnologia da informação (TI), onde os sistemas estão capacitados a economizar custos e aumentar a produtividade significativamente.

Apesar de o BPM ter uma adoção disseminada e de apresentar resultados surpreendentes, Hammer (2015), argumenta que ainda existem assuntos a serem abordados, no que diz respeito ao real gerenciamento de empresas em virtude de BPM e seu impacto nas pessoas, organizações e economias. Esse assunto vem sendo investigado constantemente o que torna o futuro desafiador em matéria de gerenciamento de processos de negócios. 


\section{Barreiras na implantação de BPM}

O foco dessa pesquisa é encontrar barreiras existentes na transição funcional. Por muito tempo visão conduziu a administração de empresas, e atualmente este método é questionado (GONÇALVES; CAMPOS, 2012). As organizações estão acostumadas a operarem num ambiente tradicional onde só reconhecem as tarefas do setor onde ocupam, e para o sucesso do projeto elas devem migrar de um ambiente habitual concentrado nas funções para um coordenado por processo. A função do BPM é melhorar os serviços para aumentar a expectativa do negócio.

Muitas vezes as empresas não estão nesta jornada por que querem, mas são obrigadas a seguir as tendências de mercado para não perderem a corrida mercadológica. Neste contexto, a alta administração busca soluções para preencher esta lacuna. De acordo com Lacerda et al. (2016), o êxito da implantação do BPM requer a colaboração de parceiros para redesenhar, discutir e eliminar as atividades que implicam retrabalho e que agregam pouco valor. Esses esforços exigem envolvimento da alta administração alinhado com os objetivos estratégicos da empresa.

A notoriedade é a importância que BPM ganhou nos últimos anos levanta uma questão de, como as organizações estão executando suas tarefas, como está sendo a transição de gerenciamento funcional para a gestão orientada a processos McCormack et al. (2009). Com isso foi realizada uma revisão da literatura visando pesquisar sobre barreiras enfrentadas no gerenciamento de processos, onde os autores realizaram estudos de casos nas empresas pesquisados e apontaram os fatores que implicam em dificuldades para a implementação do projeto. Os fatores estão expostos na Tabela 1:

\begin{tabular}{|l|l|}
\hline Barreiras encontradas na literatura & Autores \\
\hline - Falta de documentação dos processos atuais. & Lee e Dale \\
- Falta de conhecimento de processos. & $(1998)$ \\
- Insuficiência em Sistemas TI. & \\
- Recursos humanos. & \\
- Cultura organizacional. & \\
- Falta medição das prioridades, quais são importantes. & Rosemann e \\
\hline - Tecnologia e sistemas de informação. & De Bruin \\
- Cultura. & $(2005)$ \\
- Responsabilidade. & \\
- Metodologia. & Raduescu et \\
- Desempenho. & al. (2006) \\
\hline - Governança (responsabilidade nos processos de decisão). \\
- Apoio da alta administração.
\end{tabular}


- Econômico (avaliação de custo benefício).

- Falta de diretrizes de configuração do projeto.

- Falta de objetivo de modelagem.

- Falta de procedimentos de modelagem.

- Falta de metodologia de modelagem comum (Padronização).

- Falta de Infraestrutura de apoio.

- Aspectos de modelo e nível de granularidade.

- Modelo de garantia de qualidade.

- Retrabalho.

- Atualização constante.

- Gerenciamento de variantes.

- Consolidação e integração.

- Problemas relacionados aos modeladores.

- Questões relacionadas ao usuário.

- Problemas relacionados ao provedor de informação.

- Problemas relacionados a ferramentas e idiomas.

- Gestão da mudança.

- Governança.

Indulska et

- Falta de suporte de gerenciamento de topo (alta administração).

- Falta de suporte para proprietários de processos.

- Falta de experiência.

- Falta de retornos mensuráveis.

- Falta de coordenação.

- Falta de padronização.

- Falta de entendimento BPM.

- Falta de visibilidade.

- Falta de medidas de desempenho.

- Falta de progresso na maturidade do processo.

- Falta de ponto de partida clara.

- Falta de articulação coparceiros de negócios externos.

- Falta de ferramentas para BPM holística.

- Falta de capacidade tecnológica.

- Falta de monitorização processo.

- Falta de integração.

- Falta de recursos financeiros.

- Falta de tempo.

- Falta de apoio da alta administração.

- Falta de especialização em tecnologia da informação.

- Pobre conhecimento de abordagens orientadas a processos.

- Trabalho em equipe e desenvolvimento de equipe.

- Comunicação e conscientização.

al. (2006)

- Atenção ao cliente.

- Mapeamento do processo.

- Medidas de desempenho.

- Relação cliente/fornecedor.

- Gestão da mudança.

- Competência do gerenciamento de projetos. 
- Benchmarking.

- Melhoria contínua.

- Empoderamento do empregado.

- Treinamento.

- Suporte de gerenciamento superior.

- Apoio da alta administração

- Comunicação

- Governança

- Tecnologia da informação

- Requisitos de recursos. Humanos.

- Falta de colaboração.

- Falta capacidade em TI.

- Salvaguarda do conhecimento

- Falta conhecimento sobre processos.

- Falta de documentação de todos os processos.

- Expectativa negativa de futuro.

- Aceitação interna.

- Controle de poder de decisão.

- Agilidade nas decisões.

- Falta de metodologia.

- Envolvimento e percepções de pessoas envolvidas em relação ao

al. (2012) BPM.

- Um grande número de parceiros com diferentes percepções.

- O perfil de diversidade dos clientes atendidos.

- Mudanças culturais.

- Características de áreas da empresa.

- Falta de compromisso do topo.

- Falta de consenso entre as pessoas.

- Falta de estabelecimento de objetivos comuns.

- Falta de planejamento.

- Descrições de trabalho adequadas.

- Compreensão dos problemas.

- Falta de investimento.

- Necessidade de registro de aprendizagem.

- Falta de recursos financeiros.

- Falta de acessibilidade alto custo devido de implementação.

- Complexidade e falta de metodologia padrão e específico intrínseca de BPM.

- A falta de clareza em um nível estratégico.

- Falta de empresas que oferecem software e serviços de BPM.

- Falta de infraestrutura de Tecnologia da Informação.

- A falta de compreensão da tecnologia BPM e suas vantagens percebidas e / ou acreditar em BPM de desnecessidade e ineficácia na gestão de topo.

- A falta de tempo e à pressão resultante.

- Poder externo (software como um serviço ou a terceirização do projeto) implementação e / ou inadequado software BPM ou serviços para as PME. 
- Pobre conhecimento de abordagens orientadas processo e falta de educação BPM em funcionários.

- Subestimação das interrupções de fluxo de trabalho devido à mudança.

- A resistência à mudança por causa do medo da mudança tecnológica (processo de mudança e utilização consequências).

- A ausência de uma mentalidade funcional transversal entre a gestão de topo.

- A falta de recursos humanos e múltiplos papéis dos funcionários.

- Falta de apoio do Governo / envolvimento e não tomar pape de um líder orientador.

- Implementação interna pobres (por funcionários da empresa) e / ou falta de rigor metodológico na execução.

- Falta de responsabilidade e prestação de contas bem definida.

- Falta de apoio projeto de BPM na implementação e adoção (supervisores, a alta administração).

- Falta de métricas definidas comerciais e / ou protocolos de medição para o processo de avaliação de desempenho da gestão.

- Pressão das partes interessadas para preservar a estrutura firme atual.

- Alinhamento estratégico.

- Nível de investimento em TI.

Trkman

- Medição de desempenho.

- Nível de especialização do empregado.

- Mudanças organizacionais.

- Nomeação de proprietários de processos.

- Implementação de mudanças propostas.

- Uso de um sistema de melhoria contínua.

- Padronização de processos.

- Informatização.

- Automação.

- Treinamento e empoderamento de funcionários.

- Liderança.

- Comunicação.

- Responsabilidade.

- Motivação.

- Cultura .

- Alinhamento estratégico.

- Governança.

Ospsahl

- Métodos.

(2017)

- Tecnologia da informação.

- Pessoas.

- Cultura.

Tabela 1. Barreira que influenciam a implementação da gestão por processos encontrados na literatura.

Fonte: Elaborada pelo autor. 
Os resultados encontrados devem levar em consideração que, os autores realizaram estudos de caso visando encontrar barreiras que implicavam dificuldades para implantar o projeto nas organizações retratadas, de diferentes segmentos, tamanhos variados e de diversas localidades. A pesquisa foi feita levando em consideração somente BPM. Para Da Silva et al. (2012), o fornecimento de informações das tarefas de BPM e potenciais barreiras são as principais contribuições para os gerentes de projetos.

Como já vimos na tabela 1 acima, alguns fatores são recorrentes destacando-se na maioria dos estudos de casos pesquisados.

\section{METODOLOGIA}

Trata-se de uma pesquisa qualitativa onde foi baseada em uma extensa revisão bibliográfica, abordando os assuntos considerados relevantes para o objetivo proposto pelo autor. Foi elaborada uma tabela a fim de facilitar a visualização das barreiras encontradas, após, foi pedido a opinião de especialistas que prestam consultoria e coordenam projetos em empresas de várias cidades da região.

A entrevista foi composta de três perguntas estruturadas para extrair dos entrevistados as informações a respeito das barreiras encontradas na literatura. A coleta dos dados foi feita primeiramente via contato telefônico e posteriormente por email.

A opção de recorrer ao painel de especialistas (PINHEIRO et al., 2013), se deu pelo fato da necessidade de complementar a pesquisa, de maneira que possibilitasse o confronto de ideias entre os estudos de casos abordados e a percepção que os especialistas tem das empresas locais em geral.

\section{RESULTADOS}

Nesta pesquisa foram entrevistados dois especialistas. Será preservada a identificação dos mesmos, portanto serão representados pelas letras A e B. Foi elaborado um questionário onde as perguntas foram iguais para os dois especialistas.

Foram feitas as seguintes perguntas:

1. Quais os fatores mais relevantes que determinam barreira num projeto de implantação de BPM? Por que?

Especialista A: Acredito que os fatores mais relevantes estão relacionados a baixa qualificação dos colaboradores. 
Especialista B: Entendo que temos quatro mudanças significativas na implantação de um projeto de BPM, que seguem abaixo:

> Mudança interpessoal: Trabalhar o relacionamento entre os departamentos para que o ambiente se torne mais agradável e interessante;

> Mudança conceitual: Mostrar um novo conceito de realizar as tarefas de rotina de uma forma mais profissional e deixar de lado a "zona de conforto";

> Mudança cultural: Alterar a cultura de trabalho já impregnada na equipe, onde certos vícios são mantidos a anos e não são importantes para os resultados;

> Mudança estrutural: Adequação, na medida do necessário, da estrutura de trabalho dos departamentos.

2. Ter conhecimento desses fatores é relevante para o sucesso do projeto?

Especialista A: É fundamental para adequar a implantação e, se necessário, contratar novos colaboradores com capacidade de realizar os processos que conferem resultado para a empresa.

Especialista B: Sim. Principalmente nas mudanças conceituais e culturais. A empresa é o que o "dono" projetou para ela, mas isso não é uma crítica.

Nós vivemos em um país onde o espírito empreendedor é muito forte e muitas vezes as ideias aparecem e são colocadas em prática sem nenhum planejamento, mas num certo momento ganham proporção e é onde surgem os maiores problemas que se não são tratados podem levar a empresa a falência.

3. A literatura diz que os recurso para a implementação de projetos BPM muitas vezes são limitados. Portanto, alguns fatores podem ou devem ser priorizados? Se sim. Quais devem ser atacados primeiro?

Especialista A: Acredito que os pontos com maiores desperdícios ou que afetam mais os resultados finais do negócio. Gargalos, perdas ou falta de eficiências maiores devem ser atacados primeiro.

Especialista B: Sim. Devemos atacar o conhecimento do colaborador, comunicação e relacionamento:

Conhecimento do Colaborador: Muitas vezes os colaboradores executam suas atividades baseado no que foram instruídos e/ou experiências de outras empresas. Mas poucos são aqueles que buscam inovação no que fazem e isso chega a 95\% dos funcionários de uma empresa de 150 pessoas (independente do cargo que ocupam). Nos projetos que implantamos bus- 
camos trazer o conhecimento acadêmico e as boas práticas de mercado para montar o manual do processo e com isso trazer novas formas de executar a função.

Comunicação: É um problema mundial e infelizmente atrapalha e muito o andamento dos processos dentro de uma empresa. Isso quando o funcionário segura a comunicação para si e acaba prejudicando o processo seguinte. Nosso maior desafio é fazer a informação percorrer os processos e principalmente ser confiável.

> Relacionamento: As relações humanas em muitos projetos são um entrave no desenvolvimento porque as opiniões pessoais são colocadas como prioritárias ao invés das opiniões da equipe. Não é difícil encontrar retrabalhos em uma empresa em função de problemas de relacionamento entre pessoas entre processos que se relacionam (tem interface).

\section{RESULTADOS E DISCUSSÕES}

Como podemos observar, insuficiência em sistemas de TI é um dos fatores destacados. A integração da TI com os processos de negócio não podem ser negligenciados pelas organizações, é um fator fundamental para que os processos interajam entre si. É um facilitador a análise da melhoria continua nas atividades empresariais, Chong (2007), argumenta que os gerentes de projeto que operam novos sistemas em PME, podem sofrer com a falta de recursos financeiros, além de um desprendimento de tempo de funcionários indispensáveis em seus setores. A integração da TI com a alta gerência devem ocorrer desde o processo de negociação, para evitar desprendimento de tempo, e o custo relacionado com a capacidade de cada organização. Deve lembrar-se também de que existe uma discrepância entre PME e as grandes corporações em capacidade de implementar mudanças.

O apoio da alta administração, ou seja, a falta dele é um dos fatores prevalecentes para o insucesso do projeto, o problema partindo de cima consequentemente discorre para o resto da camada, segundo Raduescu et al. (2006), a alta administração é que dá sustentação em matéria de: patrocínio; compromisso; provisão de incentivo; compreensão dos objetivos; atenção as iniciativas. Além de assumir compromisso de longo prazo essencial para o gerenciamento do projeto.

A gestão da mudança é outro fator relevante. Existe resistência a mudança por parte de funcionários que ocupam a média gestão, por sentirem ameaça a sua 
posição hierárquica dentro da organização (ARTELL, 2013), existe também a necessidade de direcionar os funcionários para uma visão inovadora onde tomam mais autonomia a fim de assumirem mais responsabilidades (IMANIPOUR et al., 2012) em seus particulares processos. Não delegar responsabilidades as pessoas é um dos fatores apontado por Trkman (2010), onde alega que o treinamento e o empoderamento dos funcionários são pré-requisitos para um BPM bem-sucedido, pois funcionários treinados entregam serviços mais rapidamente, implicando em redução de custos e etc. Para ser competitiva a empresa tem que abraçar mudanças para serem mais competitivas. Deve manter uma infraestrutura necessária, uma padronização dos processos de negócios para promover mais flexibilidade para os colaboradores.

A implementação de BPM deve ser feita com base nas justificativas de qualquer projeto que seja orientado nas organizações, tais como: retorno do investimento, analise de custo-benefício e outros Elzinga et al. (1995). Não justifica ter um desprendimento de tempo, custo, além de correr risco de o projeto não apresentar resultados positivos.

\section{CONCLUSÕES}

Além de proporcionar um melhor entendimento em relação ao BPM a pesquisa ajudou a identificar vários fatores que implicam em barreiras para implementálo nas organizações. Podemos observar que gerenciar processos vai além de, somente contratar um gerente para implantar um projeto de mudança, embora este seja o primeiro passo. As características que esse projeto traz faz com que uma transformação seja proposta, desde o nível mais baixo até à alta administração. Todas as áreas devem estar envolvidas, a comunicação entre os departamentos é fundamental, muitos problemas de comunicação e de relacionamento foram observados.

Todas as áreas que apresentaram fracasso pode ser o sucesso na implantação, portanto devem ser atacados primeiro, fatores que implicam em alto custo, que comprometem o andamento do projeto devem ter prioridade. Medidas de desempenho devem ser adotadas, verificadas constantemente.

Por se tratar de um projeto complexo que envolve tecnologia da informação, não é de se estranhar que foi um dos fatores mais abordados, porem o que mais implica os desenvolvedores é o fator humano, com diferentes abordagens, tais co- 
mo, relacionamento, comunicação e muitas outras, que se elencar todos eles cobrirão a maioria das lacunas dos problemas abordados, mais isso é assunto para outro estudo.

O estudo atingiu o objetivo proposto de conhecer sobre BPM e os problemas enfrentados pelos gerentes de projetos, já conhecendo o caminho é proposto um estudo que atinja maior dimensão, com o objetivo de ampliar o horizonte da pesquisa com mais amostras para outras regiões do pais. A pesquisa foi baseada em estudos realizados na maioria fora do pais, que possui culturas diferentes, tecnologias diferentes, economias diferentes, portanto seria de fundamental importância aprofundarmos em conhecimentos da economia local.

\section{REFERÊNCIAS BIBLIOGRÁFICAS}

ARTELL, V. J. What barriers are perceived to be preventers in a serviced based organisation realising business process management?: 2013. 76 f. Dissertation (Unpublished master's thesis). University of Chester, United Kingdom, 2013.

BANDARA, W; OPSAHL, H. Developing organization-wide BPM capabilities in an SME: the approaches used, challenges and outcomes. Journal of Information Technology Teaching Cases. v. 7, n. 2. nov 2017, p. 92-113, Disponível em: $<$ https://rd.springer.com/article/10.1057/s41266-016-0014-y>. Acesso em: 26 de out de 2017.

CAMARGO JUNIOR, J, B; PIRES, S, R, I. Sistematização da implementação de outsourcing logístico por meio de práticas de gestão de projetos. Gestão \& Produção, São Carlos, v. 24, n. 2, $2017 . \quad$ p. 310-323. Disponível em: $<$ http://www.scielo.br/scielo.php?pid=S0104-

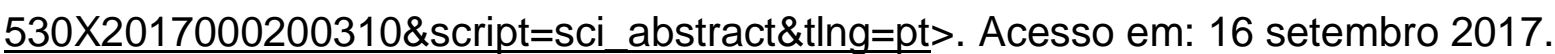

CHIAVENATO, I. Introdução à teoria geral da administração. 7. ed. Elsevier. 2003. 
CHONG, S. Business process management for SMEs: an exploratory study of implementation factors for the Australian wine industry. Journal of Information Systems and Small Business, Australia, v. 1, n. 1-2, p. 41-58, 2007. Disponível em:<https://ojs.deakin.edu.au/index.php/jissb/article/view/3 >. Acesso em: 27 de out de 2017.

DA SILVA, L.A; DAMIAN, I.P.M; DE PÁDUA, S.I.D. Process management tasks and barriers: functional to processes approach. Business Process Management Journal. Ribeirão Preto, v. 18, n. 5, 2012. P. 762-776. Disponível em: $<$ https://doi.org/10.1108/14637151211270144> . Acesso em: 18 out de 2017.

ENOKI, C.H. Gestão de processos de negócio: uma Contribuição para a Avaliação de Soluções de Business Process Management (BPM) sob a ótica da Estratégia de Operações. Tese Mestrado, USP, 2006.

ELZINGA, D, J; HOTAK, T; YEE LEE, C; BRUNER, C. Business process management: survey and methodology. leeexplore digital library. v. 42. n. 2, 1995. p. 119128. Disponível em: <http://ieeexplore.ieee.org/abstract/document/387274/>. Acesso em: 18 out de 2017.

Guia para o gerenciamento de processos de negócio corpo comum do conhecimento ABPM BPM CBOK V3.0. abpmp, 2013. Disponível em: <http://www.abpmp-br.org > . Acesso em: 16 setembro 2017

GONÇALVES, V; CAMPOS, C. Gestão de mudanças o fator humano na liderança de projetos. 1. ed. São Paulo: Brasport 2012.

HAMMER, M. What is Business Process Management?. Handbook on Business Process Management 1, v. 2, n. 1, 2015. p. 3-16. Disponível em:<https://doi.org/10.1007/978-3-642-45100-3 1>. Acesso em: 26 de out de 2017.

IMANIPOUR, N; TALEBI, K; REZAZADEH, S. Obstacles in business process manage-ment (BPM) implementation and adoption in smes. Social Science 
Electronic Publishing, Tehran, 24 jan 2012. Disponível em:<https://papers.ssrn.com/sol3/papers.cfm?abstract id=1990609 >. Acesso em: 26 de out de 2017.

INDULSKA, M., et al. Major Issues in Business Process Management: An Australian Perspective. Australasian Conference on Information Systems, n. 17, 2006, Adelaide. ACIS 2006 Proceedings.66, 2006. Elibrary, 2006. P. 1-11.

Kerzner, H. Gestao de projetos as melhores práticas. 3. ed. Porto Alegre. Bookman. 2016. 796.

Ko, R.K.L. A computer scientist's introductory guide to business process management (BPM). Crossroads, v. 15, n.4, 2009. p. 11-18. Disponível em:<http://xrds.acm.org/article.cfm?aid=1558901>. Acesso em: 12 out de 2017

LACERDA, R. T. O., et al. Research Opportunities in Business Process Management and Performance Measurement from a Constructivist View. Knowledge and Process Management. Florianopolis. v. 23, n. 1, 2016. p. 18-30. Disponível em:<http://onlinelibrary.wiley.com/doi/10.1002/kpm.1495/full>. Acesso em: 20 out 2017.

LEE. R. G; DALE, B. G. Business process management: a review and evaluation. Business Process Management Journal, Manchester, v. 4, n. 3, 1998. p. 214-225. Disponível em: https://doi.org/10.1108/14637159810224322>. Acesso em: 18 out 2017.

Mansur, R. Implementando um escritório de projetos. Rio de Janeiro: Brasport, 2007.

MCCORMACK, K., et al. A global investigation of key turning points in business process maturity. Business Process Management Journal, v. 15, n. 5, 2009. p. $792-$ 815. Disponível em:<http://www.emeraldinsight.com/doi/abs/10.1108/14637150910987946>. Acesso em: 2 nov 2017. 
MEDEIROS, A. A. Processo de definição do escopo do projeto segundo PMBOK. Revista de Ciências Gerais. Vinhedo. v. 15, n. 21, 2011. Disponível em: $<$ http://www.pgsskroton.com.br/seer/index.php/rcger/article/view/2250>. Acesso em 18 de set de 2017.

NIEHAVES, B; HENSER, J. Business Process Management Beyond Boundaries? - A Multiple Case Study Exploration of Obstacles to Collaborative BPM. System Sciences (HICSS), 2011 44th Hawaii International Conference on, n. 44, 2011, Kauai. leeexplore digital library. 2011, p. 1-13.

PINHEIRO, J. Q; FARIAS, T. M; ABE-LIMA, J. Y. Painel de especialistas e estratégia multimétodos: reflexões, exemplos, perspectivas. Psico. Porto Alegre, v. 44, n. 2, $2013 . \quad$ P. 184-192. Disponível em:<http://revistaseletronicas.pucrs.br/revistapsico/ojs/index.php/revistapsico/article/ view/11216/9635>. Acesso em: 18 de out 2017.

Project management institute (PMI). Um guia do conhecimento em gerenciamento de projetos (Guia PMBOK). 5. ed. Brasil: Editora saraiva, 2013. 616 p.

RADUESCU, C; TAN. C; JAYAGANESH, M.; SEDERA. W; MUEHLEN. M. Z; LIPPE. S. A framework of issues in large process modeling projects. ECIS 2006 Proceedings. Brisbane, v. 207. 2006. p. 1-12. Disponível em:<http://aisel.aisnet.org/ecis2006/207/>. Acesso em: 22 de out de 2017.

RAVESTEYN. P; BATENBURG. R, Surveying the critical success factors of BPMsystems implementation. Business Process Management Journal. v. 16, n. 3, 2010, p. 492-507, Disponível em: <https://doi.org/10.1108/14637151011049467>. Acesso em: 26 de out de 2017.

REZAIEA, K; OSTADIB. B; TADAYOUN, S; AGHDASI. M. Critical succes factors (CSFs) for process management projects. International Conference on industrial engineering and engineering management, 16, 2009, Beijing. leeexplore digital library. 2009. p. 100-103. 
ROSEMANN, M; DE BRUIN, T. Application of a holistic model for determining BPM maturity. BP Trends, 1 fev 2005. p. 1-21. Disponível em:<https://www.bptrends.com/application-of-a-holistic-model-for-determiningbpm-maturity/> Acesso em: 1 de out de 2017.

TRKMAN. P. The critical success factors of business process management. International Journal of Information Management. Ljubljana, v. 30, n. 2, 2010. p. 125-134. Disponível em: <https://doi.org/10.1016/j.ijinfomgt.2009.07.003>. Acesso em: 11 out de 2017.

Van der Aalst. W.M.P.Business Process Management: A Comprehensive Survey. Hindawi. Eindhoven, v. 2013, p 1-37. Disponível em: <http://dx.doi.org/10.1155/2013/507984>. Acesso em: 12 de out 2017. 